\title{
Aggregation Functions Transformed by 0 - 1 Valued Monotone Systems of Functions
}

\author{
Martin Kalina $^{(\varpi)}(\mathbb{0}$ \\ Faculty of Civil Engineering, Department of Mathematics, \\ Slovak University of Technology in Bratislava, Radlinského 11, \\ 81005 Bratislava, Slovakia \\ kalina@math.sk
}

\begin{abstract}
In the paper Jin et al. [8] the authors introduced a generalized phi-transformation of aggregation functions. This is a kind of twostep aggregation. This transformation was further developed in Jin et al. [9] into a Generalized-Convex-Sum-Transformation. A special case of the proposed Generalized-Convex-Sum-Transformation is the well-known *-product, also known as the Darsow product of copulas. This approach covers also the discrete Choquet integral. In this paper we study the monotone systems of functions, particularly the case when functions in these systems are just two-valued.
\end{abstract}

Keywords: Aggregation function $\cdot$ Copula $\cdot$ Generalized-convex-sum transformation $\cdot$ Monotone system of functions

\section{Introduction}

Jin et al. in [8] introduced a generalized $\varphi$-transformation of aggregation functions. This method is based on a so-called parametrized chain and an aggregation function $F$. The original aggregation function $A$ is transformed into $A_{\langle F, \mathbf{c}\rangle}$, where $\mathbf{c}$ is a vector-function and $F(\mathbf{c}(t))=t$. This method was modified by the same authors in [9] into a generalized-convex-sum-transformation. A special case of this generalized-convex-sum-transformation is the well-known *-product, also known as Darsow product, see [4]. As it is shown in [9], this method generalizes the discrete Choquet integral. The transformation is based on systems of monotone functions as follows (we illustrate here the transformation of binary aggregation functions)

$$
A_{\mathbb{F}}(x, y)=\int_{0}^{1} A\left(f_{x}(t), g_{y}(t)\right) \mathrm{d} t,
$$

where $\mathbb{F}$ is the pair of monotone systems of functions $\left\{f_{x}\right\}_{x \in[0,1]},\left\{g_{y}\right\}_{y \in[0,1]}$. This means that the particular choice of the pair $\mathbb{F}$ of monotone systems of functions 
$\left\{f_{x}\right\}_{x \in[0,1]},\left\{g_{y}\right\}_{y \in[0,1]}$ influences the resulting transform $A_{\mathbb{F}}$ of the (in this case) binary aggregation function $A$.

In this contribution we will study the case when the systems of functions $\left\{f_{x}\right\}_{x \in[0,1]},\left\{g_{y}\right\}_{y \in[0,1]}$ are two-valued, i.e., $f_{x}(t) \in\{0,1\}$ and $g_{y}(t) \in\{0,1\}$ for all $x, y, t \in[0,1]$.

After recalling some preliminary notions and results in Sect. 2, in Sect. 3 we provide the results of our study. Finally, conclusions are given in Sect. 4 .

\section{Preliminaries}

In this section we recall some basic definitions and known facts on aggregation functions. In the second part we provide basic idea of the generalized-convexsum-transformation that was introduced in [9].

\subsection{Basic Definitions and Known Facts}

In this contribution we will deal with ( $n$-ary) aggregation function on $[0,1]$. For more details including definitions and discussion concerning examples and properties of aggregation functions we recommend [1,2,7,10,12].

Some distinguished families of $n$-ary aggregation functions are given in the following definition.

Definition 1 ([7]). An n-ary aggregation function $A$ is said to be

(1) an $n$-ary semi-copula if $e=1$ is its neutral element,

(2) a t-norm if it is an associative and symmetric semi-copula,

(3) dual to a semi-copula if $e=0$ is a neutral element,

(4) a t-conorm if it is associative and symmetric and dual to a semi-copula,

(5) an n-ary quasi-copula if it is a 1-Lipschitz semi-copula, i.e.,

$$
\left|A\left(x_{1}, \ldots, x_{n}\right)-A\left(y_{1}, \ldots, y_{n}\right)\right| \leq \sum_{i=1}^{n}\left|x_{i}-y_{i}\right| .
$$

Definition 2 ([12]). An n-ary aggregation function $C_{n}:[0,1]^{n} \rightarrow[0,1]$ is said to be an $n$-ary copula if it is an n-ary semi-copula which is $n$-increasing, i.e., if for all $\mathbf{x}^{(0)} \in[0,1]^{n}$ and $\mathbf{x}^{(1)} \in[0,1]^{n}$ such that $\mathbf{x}^{(0)} \geq \mathbf{x}^{(1)}$ the following holds

$$
\sum_{\left(i_{1}, \ldots, i_{n}\right) \in \mathcal{I}}(-1)^{\sum_{k=1}^{n} i_{k}} C_{n}\left(x_{1}^{\left(i_{1}\right)}, \ldots, x_{n}^{\left(i_{n}\right)}\right) \geq 0,
$$

where $\mathcal{I}=\{0,1\}^{n}$.

Lemma 1 ([12]). Every copula $C$ is 1-Lipschitz.

Proposition 1 ([12]). Let $C$ be a binary copula (or a quasi-copula). Then for every $(x, y) \in[0,1]^{2}$

$$
\max (0, x+y-1) \leq C(x, y) \leq \min (x, y) .
$$


Let us remark that the functions related to the lower and upper bound occurring in inequality (2) are denoted by

$$
W(x, y)=\max (0, x+y-1), \quad M(x, y)=\min (x, y)
$$

and are called the lower and upper Fréchet-Hoeffding bounds, respectively. In the theory of t-norms the function $W$ is usually denoted by $T_{L}$ and $M$ is denoted by $T_{M}$ and are called the Łukasiewicz and minimum t-norm, respectively.

\subsection{Generalized-Convex-Sum-Transformation}

In this section we briefly recall some definitions and results from [9] explaining the construction method using transformation of a given aggregation function $A$ by an $n$-tuple of monotone systems of functions $\mathbb{F}_{n}$. The notion of a monotone system of functions is crucial in the construction method in question.

Definition 3. Let $\mathcal{F}=\left\{f_{x}\right\}_{x \in[0,1]}$ be a family of functions such that

1. for every $x \in[0,1] f_{x}:[0,1] \rightarrow[0,1]$ is a Lebesgue integrable function,

2. $f_{x_{1}} \leq f_{x_{2}}$ for $x_{1} \leq x_{2}$,

3. for all $z \in[0,1] f_{0}(z)=0$ and $f_{1}(z)=1$.

Then $\mathcal{F}$ is called a Monotone System of Functions, MSF for brevity.

Example 1. For every $x \in[0,1]$ let $f_{x}:[0,1] \rightarrow[0,1]$ and $g_{x}:[0,1] \rightarrow[0,1]$ be defined by

$$
f_{x}(t)=\left\{\begin{array}{ll}
0 & \text { for } x<1, \\
1 & \text { for } x=1,
\end{array} \quad g_{x}(t)= \begin{cases}0 & \text { for } x=0 \\
1 & \text { for } x>0\end{cases}\right.
$$

Then $\mathcal{F}=\left\{f_{x}\right\}_{x \in[0,1]}$ and $\mathcal{G}=\left\{g_{x}\right\}_{x \in[0,1]}$ are the least and the greatest Monotone Systems of Functions, respectively.

Definition 4. Let $\mathcal{F}^{(i)}=\left\{f_{x}^{(i)}\right\}_{x \in[0,1]}, i=1,2, \ldots, n$, be MSF (Definition 3). Then $\mathbb{F}_{n}=\left(\mathcal{F}^{(1)}, \mathcal{F}^{(2)}, \ldots, \mathcal{F}^{(n)}\right)$ is called an $n$-tuple of the Monotone Systems of Functions, $n$-MSF for brevity.

Lemma 2 ([9]). Let $A:[0,1]^{n} \rightarrow[0,1]$ be any Lebesgue integrable $n$-ary aggregation function and $\mathbb{F}_{n}=\left(\mathcal{F}^{(1)}, \mathcal{F}^{(2)}, \ldots, \mathcal{F}^{(n)}\right)$ be an arbitrary $n$-MSF. Let a function $A_{\mathbb{F}_{n}}:[0,1]^{n} \rightarrow[0,1]$ be given by

$$
A_{\mathbb{F}_{n}}\left(x_{1}, \ldots, x_{n}\right)=\int_{0}^{1} A\left(f_{x_{1}}^{(1)}(t), \ldots, f_{x_{n}}^{(n)}(t)\right) \mathrm{d} t
$$

where $f_{x_{1}}^{(1)} \in \mathcal{F}^{(1)}, \ldots, f_{x_{n}}^{(n)} \in \mathcal{F}^{(n)}$. Then $A_{\mathbb{F}_{n}}$ is an aggregation function.

Definition 5. An n-ary aggregation function $A_{\mathbb{F}_{n}}$ defined by formula (4), where $\mathbb{F}_{n}=\left(\mathcal{F}^{(1)}, \ldots, \mathcal{F}^{(n)}\right)$ is an $n$-tuple of monotone systems of functions, is said to be a Generalized-Convex-Sum-Transform of $A$ by $\mathbb{F}_{n}$, or a GCS-transform in short. 
Definition 6. (i) Let $\mathcal{F}=\left\{f_{x}\right\}_{x \in[0,1]}$ be an $M S F$. If $\int_{0}^{1} f_{x}(t) \mathrm{d} t=x$ is fulfilled for all $x \in[0,1]$ then $\mathcal{F}$ is called a Standard Monotone System of Functions, or SMSF for brevity.

(ii) Let $\mathcal{F}^{(i)}=\left\{f_{x}^{(i)}\right\}_{x \in[0,1]}, i=1,2, \ldots, n$, be an $S M S F$.

Then $\mathbb{F}_{n}=\left(\mathcal{F}^{(1)}, \mathcal{F}^{(2)}, \ldots, \mathcal{F}^{(n)}\right)$ is called an $n$-tuple of the Standard Monotone Systems of Functions, or n-SMSF for brevity.

Remark 1. It follows directly from Definition 3 and 6 that the set containing all the MSF and SMSF is convex.

Example 2 ([9]). Consider an SMSF $\mathcal{F}=\left\{f_{x}\right\}_{x \in[0,1]}$ such that

$$
f_{x}(t)= \begin{cases}1 & \text { for } x \in] 0,1] \text { and } t \in[0, x] \\ 0 & \text { otherwise. }\end{cases}
$$

Denote $x_{(0)}=0$ and $A\left(f_{x_{1}}(t), \ldots, f_{x_{n}}(t)\right)=A\left(\mathbf{1}_{\{(i), \ldots,(n)\}}\right)$ for $\left.\left.t \in\right] x_{(i-1)}, x_{(i)}\right]$. Then for an arbitrary $n \geq 2$, an arbitrary $n$-ary aggregation function $A$ and the $n$-tuple $\mathbb{F}_{n}=(\mathcal{F}, \ldots, \mathcal{F})$ the following holds

$$
\begin{aligned}
A_{\mathbb{F}_{n}}(\mathbf{x})= & \int_{0}^{1} A\left(f_{x_{1}}(t), \ldots, f_{x_{n}}(t)\right) \mathrm{d} t \\
= & \int_{0}^{x_{(1)}} A\left(\mathbf{1}_{\{(1), \ldots,(n)\}}\right) \mathrm{d} t+\int_{x_{(1)}}^{x_{(2)}} A\left(\mathbf{1}_{\{(2), \ldots,(n)\}}\right) \mathrm{d} t+\cdots+ \\
& +\int_{x_{(n-1)}}^{x_{(n)}} A\left(\mathbf{1}_{\{(n)\}}\right) \mathrm{d} t \\
= & x_{(1)} m_{A}(\{(1), \ldots,(n)\})+\left(x_{(2)}-x_{(1)}\right) m_{A}(\{(2), \ldots,(n)\})+\cdots+ \\
& +\left(x_{(n)}-x_{(n-1)}\right) m_{A}(\{(n)\})=\mathrm{Ch}_{m_{A}}(\mathbf{x}),
\end{aligned}
$$

where $m_{A}: 2^{\{1, \ldots, n\}} \rightarrow[0,1]$ is a capacity given by $m_{A}(E)=A\left(\mathbf{1}_{E}\right),(\cdot):$ $\{1, \ldots, n\} \rightarrow\{1, \ldots, n\}$ is any permutation satisfying $x_{(1)} \leq x_{(2)} \leq \cdots \leq x_{(n)}$, and $\mathrm{Ch}_{m_{A}}$ is the Choquet integral (see $[3,6]$ ) with respect to the capacity $m_{A}$. This fact shows that our construction method can be seen as a significant extension of the Choquet integrals.

\section{$3 \quad 0$ - 1 Valued Standard Monotone Systems of Functions}

In the rest of the paper we will consider only standard monotone systems of functions $\mathcal{F}=\left\{f_{x}\right\}_{x \in[0,1]}$ that fulfill the constraint $f_{x}(t) \in\{0,1\}$ for all $x \in[0,1]$ and $t \in[0,1]$. We will call them 0-1-valued monotone systems of functions, abbreviation 0-1-SMSF.

Let $\mathcal{F}=\left\{f_{x}\right\}_{x \in[0,1]}$ and $\mathcal{G}=\left\{g_{x}\right\}_{x \in[0,1]}$ be 0 -1-valued monotone systems of functions and $\mathbb{F}=(\mathcal{F}, \mathcal{G})$ be a pair of 0 -1-SMSF. Define $C^{\mathbb{F}}:[0,1]^{2} \rightarrow[0,1]$ by

$$
C^{\mathbb{F}}(x, y)=\int f_{x}(t) g_{y}(t) \mathrm{d} t .
$$


Let $A$ be a binary aggregation function. Then, obviously

$$
\begin{aligned}
A_{\mathbb{F}}(x, y) & =\int_{0}^{1} A\left(f_{x}(t), g_{y}(t)\right) \mathrm{d} t \\
& =\left(x-C^{\mathbb{F}}(x, y)\right) A(1,0)+\left(y-C^{\mathbb{F}}(x, y)\right) A(0,1)+C^{\mathbb{F}}(x, y) .
\end{aligned}
$$

In other words, knowing $C^{\mathbb{F}}$ we know the result of the GCS-transform $A_{\mathbb{F}}$ of $A$.

We will focus our attention only to binary aggregation functions and their GCS-transforms.

Proposition 2. Let $\mathcal{F}=\left\{f_{x}\right\}_{x \in[0,1]}$ and $\mathcal{G}=\left\{g_{x}\right\}_{x \in[0,1]}$ be arbitrary 0 -1-SMSF and $\mathbb{F}=(\mathcal{F}, \mathcal{G})$. Then, $C^{\mathbb{F}}$ defined by formula $(6)$ is a copula.

Proof. Directly by Definitions 3 and 6 we have that $C^{\mathbb{F}}$ is a semi-copula. Let us prove the two-increasingness. Assume $x_{1} \geq x_{2}$ and $y_{1} \geq y_{2}$ be arbitrary elements of $[0,1]$. Then for all $t \in[0,1]$ the following holds

$$
f_{x_{1}}(t) \geq f_{x_{2}}(t), \quad g_{y_{1}}(t) \geq g_{y_{2}}(t)
$$

These imply

$$
\begin{aligned}
& C^{\mathbb{F}}\left(x_{1}, y_{1}\right)-C^{\mathbb{F}}\left(x_{2}, y_{1}\right)=\int_{0}^{1}\left(f_{x_{1}}(t)-f_{x_{2}}(t)\right) g_{y_{1}}(t) \mathrm{d} t \\
& C^{\mathbb{F}}\left(x_{1}, y_{2}\right)-C^{\mathbb{F}}\left(x_{2}, y_{2}\right)=\int_{0}^{1}\left(f_{x_{1}}(t)-f_{x_{2}}(t)\right) g_{y_{2}}(t) \mathrm{d} t .
\end{aligned}
$$

Formulae (8) and (9) imply

$$
\begin{aligned}
& \left(C^{\mathbb{F}}\left(x_{1}, y_{1}\right)-C^{\mathbb{F}}\left(x_{2}, y_{1}\right)\right)-\left(C^{\mathbb{F}}\left(x_{1}, y_{2}\right)-C^{\mathbb{F}}\left(x_{2}, y_{2}\right)\right) \\
= & \int_{0}^{1}\left(f_{x_{1}}(t)-f_{x_{2}}(t)\right)\left(g_{y_{1}}(t)-g_{y_{2}}(t) \mathrm{d} t,\right.
\end{aligned}
$$

and by inequalities (7),

$$
\int_{0}^{1}\left(f_{x_{1}}(t)-f_{x_{2}}(t)\right)\left(g_{y_{1}}(t)-g_{y_{2}}(t) \mathrm{d} t \geq 0 .\right.
$$

Definition 7. Let $\mathcal{F}=\left\{f_{x}\right\}_{x \in[0,1]}$ and $\mathcal{G}=\left\{g_{x}\right\}_{x \in[0,1]}$ be arbitrary 0-1-SMSF and $\mathbb{F}=(\mathcal{F}, \mathcal{G})$. Then we denote $C^{\mathbb{F}}$ the copula given by Eq. (6) and we say that $C^{\mathbb{F}}$ is generated by $\mathbb{F}$.

The following example illustrates some 0-1-SMSF and the copulas they generate.

Example 3. Denote $\tilde{\mathcal{F}}=\left\{f_{x}\right\}_{x \in[0,1]}$ where $f_{x}$ are given by formula (5). Further, set $\mathcal{G}_{1}=\left\{{ }^{1} g_{x}\right\}_{x \in[0,1]}, \mathcal{G}_{2}=\left\{{ }^{2} g_{x}\right\}_{x \in[0,1]}, \mathcal{G}_{3}=\left\{{ }^{3} g_{x}\right\}_{x \in[0,1]}, \mathcal{G}_{4}=\left\{{ }^{4} g_{x}\right\}_{x \in[0,1]}$, 
$\mathcal{G}_{5}=\left\{{ }^{5} g_{x}\right\}_{x \in[0,1]}$, where ${ }^{i} g_{x}$ are given by formulae (10), (11), (12), (13) and (14), respectively:

$$
\begin{aligned}
& { }^{1} g_{x}(t)= \begin{cases}1 & \text { if } t \in\left[0, \frac{x}{2}\right] \cup\left[1-\frac{x}{2}, 1\right], \\
0 & \text { otherwise, }\end{cases} \\
& { }^{2} g_{x}(t)= \begin{cases}1 & \text { if } t \in\left[\frac{1-x}{2}, \frac{1+x}{2}\right] \\
0 & \text { otherwise }\end{cases} \\
& { }^{3} g_{x}(t)= \begin{cases}1 \text { for: } & x \leq \frac{1}{2} \text { and } t \in[0, x], \\
& x \in] \frac{1}{2}, \frac{3}{4}\left[\text { and } t \in\left[0, \frac{1}{2}\right] \cup\left[\frac{3}{2}-x, 1\right],\right. \\
& t \geq \frac{3}{4} \text { and } t \in\left[0, x-\frac{1}{4}\right] \cup\left[\frac{3}{4}, 1\right], \\
0 & \text { otherwise, }\end{cases} \\
& { }^{4} g_{x}(t)= \begin{cases}1 \text { for: } & x \leq \frac{1}{2} \text { and } t \in\left[0, x^{2}\right] \cup\left[1-x+x^{2}, 1\right], \\
& x \in] \frac{1}{2}, \frac{3}{4}\left[\text { and } t \in\left[0, \frac{1}{4}\right] \cup\left[\frac{3}{4}, 1\right] \cup\left[1-x, \frac{1}{2}\right],\right. \\
& t \geq \frac{3}{4} \text { and } t \in\left[0, x-\frac{1}{4}\right] \cup\left[\frac{3}{4}, 1\right], \\
& \text { otherwise, }\end{cases} \\
& { }^{5} g_{x}(t)= \begin{cases}1 & \text { for } t \in\left[0, \frac{x}{2}\right] \cup\left[\frac{1}{2}, \frac{1}{2}(2-x)\right], \\
0 & \text { otherwise. }\end{cases}
\end{aligned}
$$
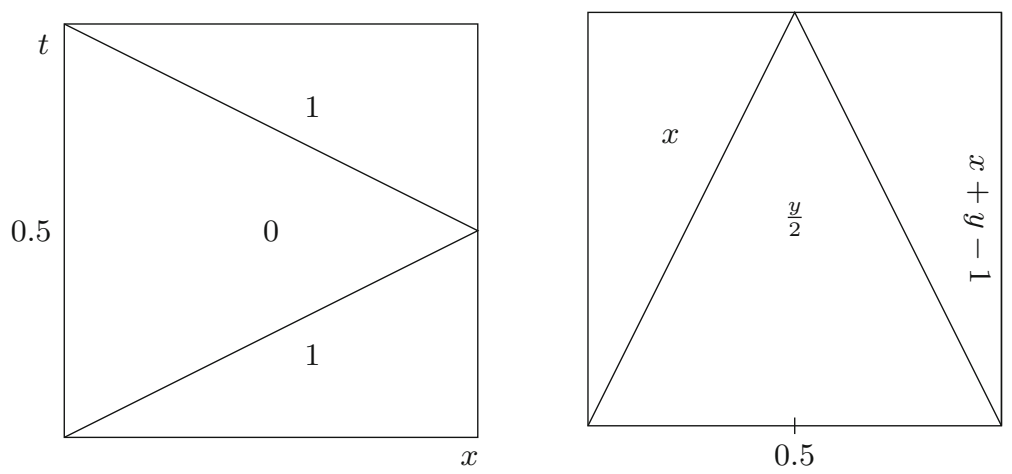

Fig. 1. Left the layout of $\mathcal{G}_{1}$, right the copula $C^{\mathbb{F}^{(1)}}$ where $\mathbb{F}^{(1)}=\left(\tilde{\mathcal{F}}, \mathcal{G}_{1}\right)$

The copula sketched in the right part of Fig. 1 is the so-called tent copula (see, e.g., [4]).

The following lemma is straightforward.

Lemma 3. Let $\mathcal{F}=\left\{f_{x}\right\}_{x \in[0,1]}$ and $\mathcal{G}=\left\{g_{x}\right\}_{x \in[0,1]}$ be arbitrary 0-1-SMSF. Then 

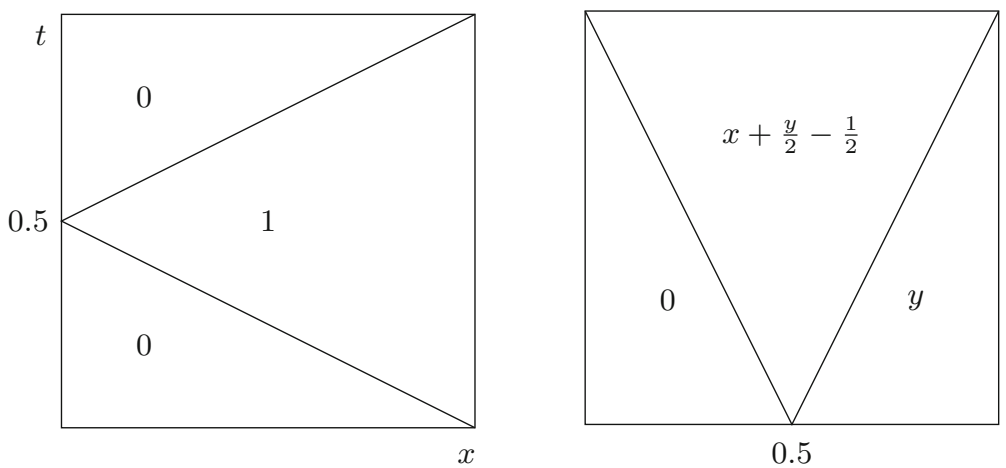

Fig. 2. Left the layout of $\mathcal{G}_{2}$, right the copula $C^{\mathbb{F}^{(2)}}$ where $\mathbb{F}^{(2)}=\left(\tilde{\mathcal{F}}, \mathcal{G}_{2}\right)$.
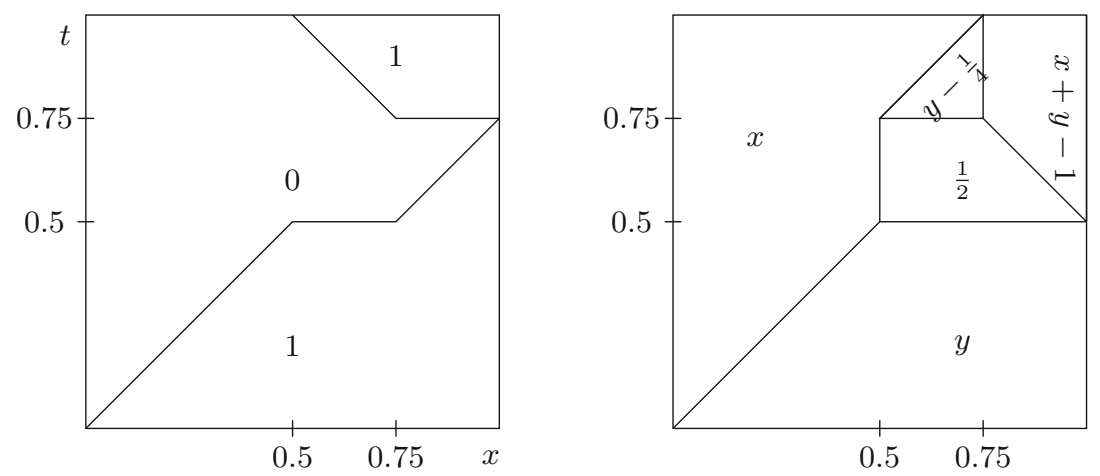

Fig. 3. Left the layout of $\mathcal{G}_{3}$, right the copula $C^{\mathbb{F}^{(3)}}$ where $\mathbb{F}^{(3)}=\left(\tilde{\mathcal{F}}, \mathcal{G}_{3}\right)$

(a) $C^{\mathbb{F}}=M$ for $\mathbb{F}=(\mathcal{F}, \mathcal{F})$,

(b) if $\mathbb{F}=(\mathcal{F}, \mathcal{G})$ generates a copula $C^{\mathbb{F}}$ then $\overline{\mathbb{F}}=(\mathcal{G}, \mathcal{F})$ generates the copula $C^{\overline{\mathbb{F}}}$ such that $C^{\overline{\mathbb{F}}}(x, y)=C^{\mathbb{F}}(y, x)$,

(c) $C^{\hat{\mathbb{F}}}=W$ for $\hat{\mathbb{F}}=\left(\mathcal{F}, \mathcal{F}^{d}\right)$, where $\mathcal{F}^{d}=\left\{f_{x}^{d}\right\}_{x \in[0,1]}$ and $f_{x}^{d}(t)=1-f_{1-x}(t)$.

Proposition 3. Let $\tilde{\mathcal{F}}$ be the 0-1-SMSF defined by formula (5). There exists a pair of 0 -1-SMSF $\mathbb{G}=\left(\mathcal{G}^{(1)}, \mathcal{G}^{(2)}\right)$ and the copula $C^{\mathbb{G}}$ generated by $\mathbb{G}$ such that for arbitrary 0 -1-SMSF $\mathcal{F}=\left\{f_{x}\right\}_{x \in[0,1]}$ and the pair of 0-1-SMSF $\mathbb{F}=(\tilde{\mathcal{F}}, \mathcal{F})$ we have

$$
C^{\mathbb{G}} \neq C^{\mathbb{F}} .
$$

Proof. Choose $(x, y) \in[0,1]^{2}$. Then

$$
C^{\mathbb{F}}(x, y)=\int_{0}^{x} f_{y}(t) \mathrm{d} t .
$$

There are three possibilities for the result of $C^{\mathbb{F}}(x, y)$. 

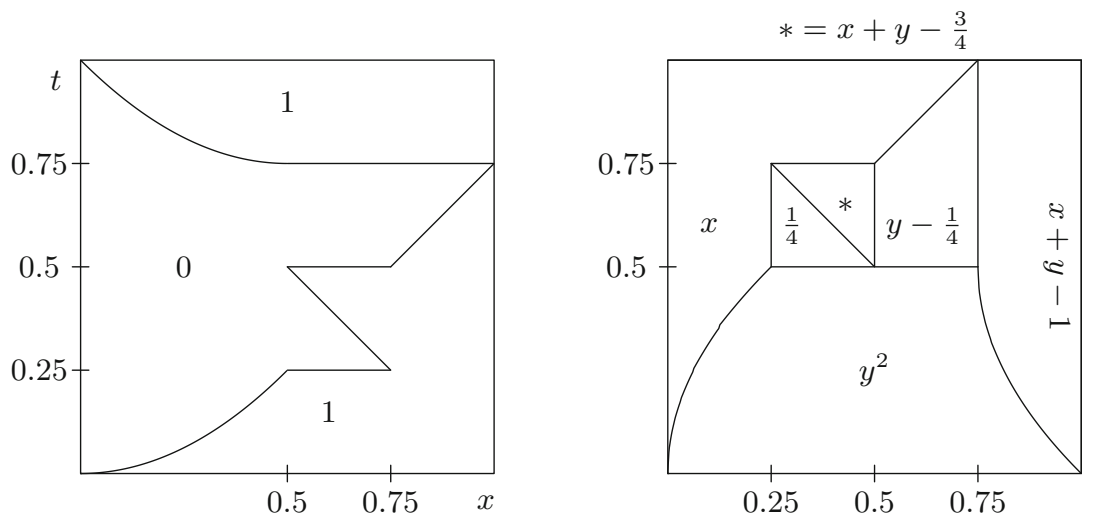

Fig. 4. Left the layout of $\mathcal{G}_{4}$, right the copula $C^{\mathbb{F}^{(4)}}$ where $\mathbb{F}^{(4)}=\left(\tilde{\mathcal{F}}, \mathcal{G}_{4}\right)$
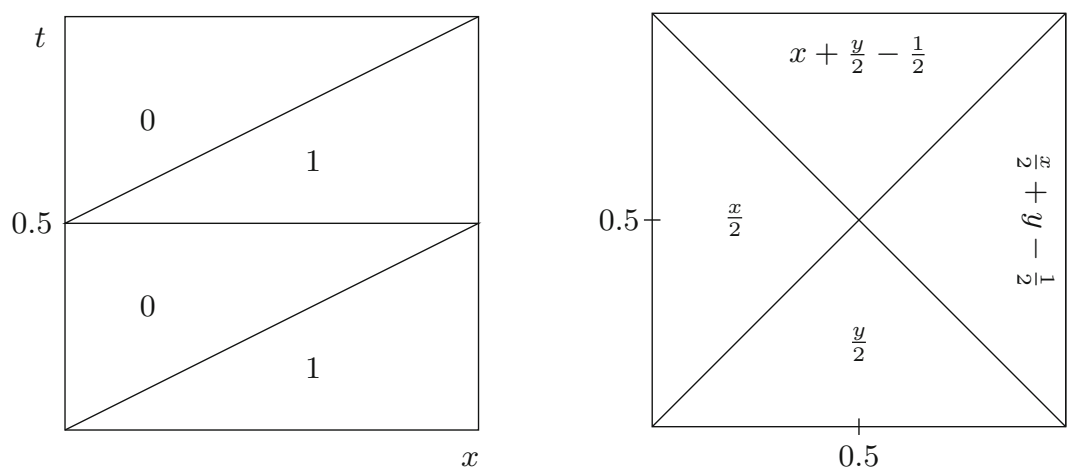

Fig. 5. Left the layout of $\mathcal{G}_{5}$, right the copula $C^{\mathbb{G}}$ where $\mathbb{G}=\left(\mathcal{G}_{1}, \mathcal{G}_{5}\right)$

1. $g_{y}(t)=0$ almost everywhere on the interval $[0, x]$, i.e., $\lambda\left(\left\{t \in[0, x] ; g_{y}(t) \neq\right.\right.$ $0\})=0$, where $\lambda$ is the Lebesgue measure. In this case $C^{\mathbb{F}}(x, y)=0$.

2. There exists $\varphi(y) \in[0,1]$ such that $f_{y}(t)=1$ almost everywhere on the interval $[0, \varphi(y)]$ and, if $x>\varphi(y) f_{y}(t)=0$ almost everywhere on $[\varphi(y), x]$. Then

$$
C^{\mathbb{F}}(x, y)= \begin{cases}x & \text { if } x \leq \varphi(y), \\ \varphi(y) & \text { if } x>\varphi(y) .\end{cases}
$$

3. There exists a countable (finite or infinite) system of pairwise disjoint intervals $\left\{\left[a_{i}, b_{i}\right]\right\}_{i \in I}$ such that $\left[a_{i}, b_{i}\right] \cap[0, x] \neq \emptyset, f_{y}(t)=1$ almost everywhere for $t \in \bigcup_{i \in I}$ and $f_{y}(t)=0$ almost everywhere for $t \in[0, x] \backslash \bigcup_{i \in I}$. There are two possibilities:

$-b_{i} \leq x$ for all $i \in I$, then

$$
C^{\mathbb{F}}(x, y)=\sum_{i \in I}\left(b_{i}-a_{i}\right)
$$


- there exists $j \in I$ such that $b_{j}>x$, then

$$
C^{\mathbb{F}}(x, y)=\sum_{i \in I}\left(b_{i}-a_{i}\right)-\left(b_{j}-x\right) .
$$

This gives the following - setting $\mathcal{G}^{(1)}=\mathcal{G}_{1}$ and $\mathcal{G}^{(2)}=\mathcal{G}_{5}$, where $\mathcal{G}_{1}$ and $\mathcal{G}_{5}$ are defined by formulae (10) and (14), respectively, and $\mathbb{G}=\left(\mathcal{G}^{(1)}, \mathcal{G}^{(2)}\right.$ ) (see also Fig. 5), then the copula $C^{\mathcal{G}}$ cannot be generated by any pair of 0-1-SMSF $\mathbb{F}=(\tilde{\mathcal{F}}, \mathcal{F})$.

Remark 2. Analysing the proof of Proposition 3 we see that if a copula has a component that is non-linear in the first variable, then it cannot be generated by a pair of 0 -1-SMSF $(\tilde{\mathcal{F}}, \mathcal{F})$ where $\tilde{\mathcal{F}}$ is the 0 -1-SMSF defined by formula $(5)$. Another consequence that can be derived is that the copula $C^{\mathbb{G}}$ sketched on Fig. 5 cannot be generated by a pair of 0-1-SMSF $(\tilde{\mathcal{F}}, \mathcal{F})$ nor by $(\mathcal{F}, \tilde{\mathcal{F}})$.

A characterization by the second mixed partial derivatives of copulas generated by pairs of 0-1-SMSF is contained in the following proposition.

Proposition 4. Let $\mathbb{F}$ be a pair of 0-1-SMSF and $C^{\mathbb{F}}$ the copula generated by F. Then

$$
\frac{\partial^{2} C^{\mathbb{F}}}{\partial x \partial y}(x, y)=0
$$

for all $(x, y) \in[0,1]^{2}$ where the second mixed partial derivative exists.

Durante et al. [5] have shown that if formula (15) holds for a copula $C^{\mathbb{F}}$ then $C^{\mathbb{F}}$ still may have a density.

Finally, we show how we can construct an arbitrary shuffle of $M$ by a pair of 0-1-SMSF. The family of all shuffles of min is very important, since, as it is proven in [11], this family is dense in the system of all bivariate copulas.

A geometrical visualisation of a shuffle of min is quite straightforward. We choose a natural number $n>1$, a system of nods $0=a_{0}<a_{1}<\cdots<a_{n}=1$ and cut the minimum copula parallel to the $y$-axis into $n$ strips using those nods. Then we shuffle the strips (this means we choose a permutation $\Pi$ : $\{1,2, \ldots, n\} \rightarrow\{1,2, \ldots, n\})$ and paste them together in the permuted order. If we denote $b_{i}=a_{i}-a_{i-1}$ for $i \in\{1,2, \ldots, n\}$ then choosing a permutation $\Pi:\{1,2, \ldots, n\} \rightarrow\{1,2, \ldots, n\}$, a shuffle of min is given by $S=\left\langle n,\left(b_{i}\right)_{i=1}^{n}, \Pi\right\rangle$.

Example 4. Set $n=5$, the permutation $\Pi$ by $(2,1,4,3,5)$ and the nodes are given by $(0,0.3,0.4,0.7,0.9,1)$. Then the corresponding 0 -1-SMSF $\mathcal{G}_{6}=\left\{{ }^{6} g_{x}\right\}_{x \in[0,1]}$ is given by formula (16) and the shuffle copula is then generated by the pair of $0-1-\mathrm{SMSF} \mathbb{F}_{6}=\left(\tilde{\mathcal{F}}, \mathcal{G}_{6}\right)$ and displayed in Fig. 6. 

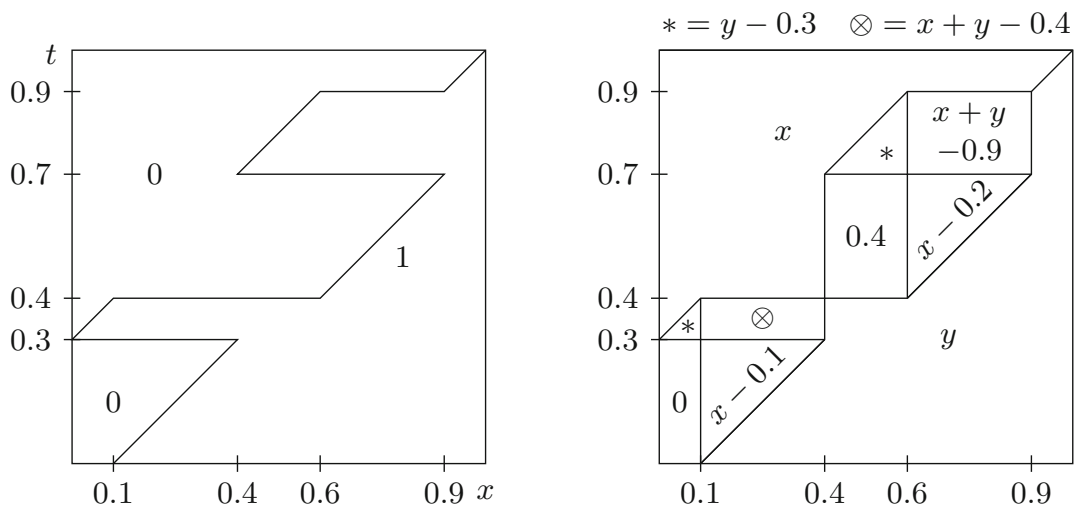

Fig. 6. Left the layout of $\mathcal{G}_{6}$, right the copula $C^{\mathbb{F}^{(6)}}$ where $\mathbb{F}^{(6)}=\left(\tilde{\mathcal{F}}, \mathcal{G}_{6}\right)$

The explicit formula for 0-1-SMSF $\mathcal{G}_{6}=\left\{{ }^{6} g_{x}\right\}_{x \in[0,1]}$ is given by

$$
{ }^{6} g_{x}(t)= \begin{cases}1 \text { for: } & x \in[0,0.1] \text { and } t \in[0.3, x+0.3], \\ & x \in[0.1,0.4] \text { and } t \in[0, x-0.1] \cup[0.3,0.4], \\ & x \in[0.4,0.6] \text { and } t \in[0,0.4] \cup[0.7, x+0.3], \\ & x \in[0.6,0.9] \text { and } t \in[0, x-0.2] \cup[0.7,0.9], \\ & x \in[0.9,1] \text { and } t \in[0, x], \\ 0 & \text { otherwise. }\end{cases}
$$

Shuffles of min are in [11] described in a more general way than we have illustrated by Example 4. Namely, the shuffles can be combined with flips (a flip of the minimum copula $M$ is $W$ ). This combination is sketched in Fig. 3. The following proposition gives a characterization of all shuffles (possibly combined with flips) of min as special cases of copulas generated by a pair of 0-1-SMSF. In this case a shuffle of min is given by $\left\langle n,\left(b_{i}\right)_{i=1}^{n}, \Pi,\left(m_{i}\right)_{i=1}^{n}\right\rangle$, where $b_{i}$ denotes the width of the $i$-th strip, $\Pi$ is a permutation of $\{1,2, \ldots, n\}$ and $m_{i}=1(m=0)$ if we use the flip (if we do not use the flip) in the square $\left[a_{i-1}, a_{i}\right]^{2}$, where $a_{i}=\sum_{j=1}^{i} b_{j}$. We skip the proof of Proposition 5 since the construction there is just a generalization of formulae (12) and (16).

Proposition 5. Set $n>1$ a natural number, for $i \in\{1, \ldots, n\}$ let $b_{i}>0$ be such that $\sum_{i=1}^{n} b_{i}=1, m_{i} \in\{0,1\}$ and $\Pi:\{1, \ldots, n\} \rightarrow\{1, \ldots, n\}$ be a permutation.

Denote $a_{0}=\tilde{a}_{0}=0$ and for $i \in\{1, \ldots, n\} a_{i}=\sum_{j=1}^{i} b_{j}$ and $\tilde{a}_{i}=\sum_{j=1}^{i} b_{\Pi^{-1}(j)}$. The shuffle of min given by $\left\langle n,\left(b_{i}\right)_{i=1}^{n}, \Pi,\left(m_{i}\right)_{i=1}^{n}\right\rangle$, is generated by the pair of 0-1-SMSF $\mathbb{F}=(\tilde{\mathcal{F}}, \mathcal{H})$, where $\mathcal{H}=\left\{h_{x}\right\}_{x \in[0,1]}$ is defined by 


$$
h_{x}(t)=\left\{\begin{array}{l}
1 \quad \text { for } x \in\left[0, \tilde{a}_{1}\right] \text { and } t \in A_{1}, \\
\quad x \in\left[\tilde{a}_{i-1}, \tilde{a}_{i}\right] \text { and } t \in A_{i} \cup \bigcup_{j=1}^{i-1}\left[\tilde{a}_{j-1}, \tilde{a}_{j}\right], \text { for } 2 \leq i \leq n, \\
0 \quad \text { otherwise, }
\end{array}\right.
$$

where, for $i \in\{1,2, \ldots, n\}$,

$$
A_{i}= \begin{cases}{\left[a_{\Pi}^{-1}(i)+\tilde{a}_{i-1}-x, a_{\Pi^{-1}(i)}\right]} & \text { if } m=1 \\ {\left[a_{\Pi}^{-1}(i)-1, x-\left(a_{\Pi^{-1}(i)}-\tilde{a}_{i}\right)\right]} & \text { if } m=0 .\end{cases}
$$

\section{Conclusion}

This paper contributes to a study of Generalized-Convex-Sum-Transformation of (binary) aggregation functions. Particularly, we have studied copulas that can be generated by pairs of 0-1-SMSF (see formula (6)). Though, the 0-1-SMSF $\tilde{\mathcal{F}}$ given by formula (5) is, in a sense, a basic 0-1-SMSF, when we like to generate all possible copulas by pairs of 0-1-SMSF, it is not sufficient to consider only those pairs where one of the $0-1-\mathrm{SMSF}$ is $\tilde{\mathcal{F}}$. We have also shown that every shuffle of the minimum copula (possibly combined with flips) can be generated by a pair of 0-1-SMSF and in Proposition 5 we have written down an explicit formula for such 0-1-SMSF.

Acknowledgements. The work on this paper has been supported from the Science and Technology Assistance Agency under the contract no. APVV-18-0052, and by the Slovak Scientific Grant Agency VEGA no. 1/0006/19 and 2/0142/20.

\section{References}

1. Beliakov, G., Bustince Sola, H., Calvo Sánchez, T.: A Practical Guide to Averaging Functions. SFSC, vol. 329. Springer, Cham (2016). https://doi.org/10.1007/9783-319-24753-3

2. Beliakov, G., Pradera, A., Calvo, T.: Aggregation Functions: A Guide for Practitioners. Springer, Heidelberg (2007). https://doi.org/10.1007/978-3-54073721-6

3. Choquet, G.: Theory of capacities. Ann. Inst. Fourier 5, 131-295 (1953-1954)

4. Darsow, W.F., Nguyen, B., Olsen, T.E.: Copulas and Markov processes. Ill. J. Math. 36(4), 600-642 (1992)

5. Durante, F., Sánchez, J.F., Sempi, C.: A note on the notion of singular copula. Fuzzy Sets Syst. 211, 120-122 (2013). https://doi.org/10.1016/j.fss.2012.04.005

6. Grabisch, M.: Fuzzy integral in multicriteria decision making. Fuzzy Sets Syst. 69(3), 279-298 (1995)

7. Grabisch, M., Marichal, J.L., Mesiar, R., Pap, E.: Aggregation Functions. Cambridge University Press, Cambridge (2009)

8. Jin, L., Mesiar, R., Kalina, M., Špirková, J., Borkotokey, S.: Generalized phitransformations of aggregation functions. Fuzzy Sets Syst. 372, 124-141 (2019). https://doi.org/10.1016/j.fss.2018.09.016 
9. Jin, L., Mesiar, R., Kalina, M., Špirková, J., Borkotokey, S.: New transformations of aggregation functions based on monotone systems of functions. Int. J. Approximate Reasoning 118, 79-95 (2020). https://doi.org/10.1016/j.ijar.2019.12.004

10. Klement, E.P., Mesiar, R., Pap, E.: Triangular Norms. Springer, Heidelberg (2000). https://doi.org/10.1007/978-94-015-9540-7

11. Mikusinski, P., Sherwood, H., Taylor, M.D.: Shuffles of min. Stochastica XIII-1, 61-74 (1992)

12. Nelsen, R.B.: An Introduction to Copulas. Springer Series in Statistics. Springer, New York (2006). https://doi.org/10.1007/0-387-28678-0 